\title{
Asymptotic BEP and SEP of Quadratic Diversity Combining Receivers in Correlated Ricean Fading, Non-Gaussian Noise, and Interference
}

\author{
Ali Nezampour ${ }^{\dagger}$, Amir Nasri ${ }^{\dagger}$, Robert Schober ${ }^{\dagger}$, and Yao Ma ${ }^{\dagger \dagger}$ \\ ${ }^{\dagger}$ The University of British Columbia \\ E-mail: $\{$ alinezam, amirn, rschober\}@ece.ubc.ca \\ i† lowa State University, Ames \\ E-mail: mayao@iastate.edu
}

\begin{abstract}
In this paper, we study the asymptotic behavior of the bit-error probability (BEP) and the symbolerror probability (SEP) of quadratic diversity combining schemes such as coherent maximum-ratio combining (MRC), differential equal-gain combining (EGC), and noncoherent combining (NC) in correlated Ricean fading and non-Gaussian noise, which in our definition also includes interference. We provide simple and easy-to-evaluate asymptotic BEP and SEP expressions which show that at high signal-to-noise ratios (SNRs) the performance of the considered combining schemes depends on certain moments of the noise and interference impairing the transmission. We derive general rules for calculation of these moments and we provide closed-form expressions for the moments of several practically important types of noise such as spatially dependent and spatially independent Gaussian mixture noise, correlated synchronous and asynchronous co-channel interference, and correlated Gaussian interference. From our asymptotic results we observe that (a) the asymptotic performance loss of binary frequency-shift keying (BFSK) with NC compared to binary phase-shift keying (BPSK) with MRC is always $6 \mathrm{~dB}$ independent of the type of noise and the number of diversity branches, (b) the asymptotic performance loss of differential EGC compared to MRC is always $3 \mathrm{~dB}$ for additive white Gaussian noise but depends on the number of diversity branches and may be larger or smaller than $3 \mathrm{~dB}$ for other types of noise, and (c) not only fading correlation but also noise correlation negatively affects the performance of quadratic diversity combiners.
\end{abstract}




\section{Introduction}

In recent years the performance analysis of wireless communication systems impaired by fading and noise has received considerable attention, cf. e.g. [1] and references therein. In particular, for fading channels impaired by additive white Gaussian noise (AWGN) simple closed-form expressions for the symbol error probability (SEP) at high signal-to-noise ratio (SNR) have been developed, cf. [2]-[7]. These asymptotic expressions are very useful for communication system design as they reveal the effects of the modulation scheme, the diversity combining scheme, and the channel parameters on system performance.

In practice, wireless communication systems are often not only impaired by AWGN but also by non-Gaussian noise and interference ${ }^{1}$. Examples of non-Gaussian noise include co-channel and adjacent channel interference [8], impulsive noise [9], and ultra-wideband (UWB) interference [10]. Recently, the authors have provided a unified asymptotic SEP analysis of coherent equal gain combining (EGC) and coherent selection combining (SC) which is applicable to many different types of noise and independent identically distributed (i.i.d.) diversity branches [11]. Unfortunately, the approach used in [11] cannot be extended to the important class of quadratic diversity combining techniques which include coherent maximum ratio combining (MRC), differential EGC, and noncoherent combining (NC).

In this paper, we present a novel powerful framework for analyzing quadratic diversity combining techniques in the high SNR regime when the received signal is impaired by correlated Ricean fading and general non-Gaussian noise. Since the only assumption that we make on the noise is that all of its moments exist, our results are applicable to a large number of practical scenarios. The resulting asymptotic bit error probability (BEP) and SEP expressions are surprisingly simple and easy to evaluate and only require the calculation of certain noise moments. We show how these noise moments can be efficiently obtained for several practically relevant types of noise including spatially dependent and spatially independent $\epsilon$-mixture noise, synchronous and asynchronous co-channel interference, spatially correlated Gaussian interference, and UWB interference.

The remainder of this paper is organized as follows. In Section 2, some definitions and the

\footnotetext{
${ }^{1}$ To simplify our notation, in the following, "noise" refers to any additive impairment of the received signal, i.e., our definition of noise also includes what is commonly referred to as "interference".
} 
considered signal model are introduced. In Section 3, general expressions for the asymptotic BEPs and SEPs of MRC, differential EGC, and NC are derived. Techniques for calculation of the moments of relevant types of noise are provided in Section 4. In Section 5, some examples are given to illustrate the application of the obtained analytical results, and conclusions are drawn in Section 6.

\section{Preliminaries}

After introducing some definitions and notations, we present the considered signal and channel model in this section.

\subsection{Some Definitions and Notations}

Notation: In this paper, bold lower case letters $\boldsymbol{x}$ and bold upper case letters $\boldsymbol{X}$ denote vectors and matrices, respectively. Furthermore, $\mathcal{E}\{\cdot\}, \operatorname{Pr}\{A\},[\cdot]^{T},(\cdot)^{*},[\cdot]^{H},\|\cdot\|$, and $\operatorname{det}(\cdot)$ denote statistical expectation, the probability of event $A$, transposition, complex conjugation, Hermitian transposition, the $L_{2}$-norm of a vector, and the determinant of a matrix, respectively. We also use the following definitions and functions: $(2 N-1) ! ! \triangleq 1 \cdot 3 \cdot \ldots \cdot(2 N-1),(2 N) ! ! \triangleq 2 \cdot 4 \cdot \ldots \cdot 2 N,\left(\begin{array}{c}L \\ k_{1}, \ldots, k_{L}\end{array}\right) \triangleq \frac{L !}{k_{1} ! \ldots k_{L} !}$, and $\boldsymbol{I}_{X}, \mathbf{0}_{X}$, and ${ }_{1} F_{1}(\cdot, \cdot ; \cdot)$ denote the $X \times X$ identity matrix, the $X$-dimensional all-zeros column vector, and the Kummer confluent hypergeometric function, respectively. $A \doteq B$ and $A \leq B$ mean that $A$ is asymptotically (for high SNR), respectively, equal to and smaller than or equal to $B$. Finally, $\Phi(s)=\mathcal{L}\{p(x)\} \triangleq \int_{-\infty}^{\infty} p(x) e^{-s x} \mathrm{~d} x$ denotes the Laplace transform of $p(x)$.

Moments: We define the $N$ th moment of the real random variable (RV) $|x|^{2}$ as $M_{x}(N) \triangleq \mathcal{E}\left\{|x|^{2 N}\right\}$, where $x$ is a complex RV. Similarly, for a complex random vector variable (RVV) $\boldsymbol{x}$ we define the $N$ th moment of $\|\boldsymbol{x}\|^{2}$ as $M_{\boldsymbol{x}}(N) \triangleq \mathcal{E}\left\{\|\boldsymbol{x}\|^{2 N}\right\}$. We note that $M_{\boldsymbol{x}}(0)=1$ and $M_{\boldsymbol{x}}(1)$ is the sum of the powers of the elements of $\boldsymbol{x}$.

Combining gain and diversity gain: For high SNRs the SEP in flat fading channels can be approximated by $[2,4]$

$$
\mathrm{SEP} \doteq\left(G_{c} \bar{\gamma}\right)^{-G_{d}}
$$


where $\bar{\gamma}$ denotes the average SNR, and $G_{c}$ and $G_{d}$ are referred to as the combining gain ${ }^{2}$ and the diversity gain, respectively.

\section{$2.2 \quad$ Signal Model}

Assuming for the moment a linear modulation format ${ }^{3}$, the signal $r_{l}[k]$ received in the $l$ th diversity branch in the $k$ th symbol interval can be modeled in equivalent complex baseband representation as

$$
r_{l}[k]=\sqrt{\bar{\gamma}} h_{l} b[k]+n_{l}[k], \quad 1 \leq l \leq L
$$

where $L, h_{l}, b[k]$, and $n_{l}[k]$ denote the number of diversity branches, the fading gain of the $l$ th branch, the transmitted symbol, and the noise in the lth diversity branch, respectively. Using vector notation, Eq. (2) can be rewritten as

$$
\boldsymbol{r}[k]=\sqrt{\bar{\gamma}} \boldsymbol{h} b[k]+\boldsymbol{n}[k]
$$

where $\boldsymbol{r}[k] \triangleq\left[r_{1}[k] r_{2}[k] \ldots r_{L}[k]\right]^{T}, \boldsymbol{h} \triangleq\left[h_{1} h_{2} \ldots h_{L}\right]^{T}$, and $\boldsymbol{n}[k] \triangleq\left[n_{1}[k] n_{2}[k] \ldots n_{L}[k]\right]^{T}$. To simplify our notation, in the following, we will drop the argument $k$ whenever this is possible without loss of generality. The transmitted symbols $b \in \mathcal{A}$ are normalized to $\mathcal{E}\left\{|b|^{2}\right\}=1$ and are take from signal constellation $\mathcal{A}$, cf. Section 3 .

The channel vector $\boldsymbol{h}$ is Gaussian distributed with mean $\boldsymbol{\mu}_{h} \triangleq \mathcal{E}\{\boldsymbol{h}\}$ and covariance matrix $\boldsymbol{C}_{h h} \triangleq \mathcal{E}\left\{\left(\boldsymbol{h}-\boldsymbol{\mu}_{h}\right)\left(\boldsymbol{h}-\boldsymbol{\mu}_{h}\right)^{H}\right\}$. We assume that $\boldsymbol{C}_{h h}$ has full rank $L$ and define the Ricean factor of the $l$ th branch as $K_{l} \triangleq\left|\mu_{l}\right|^{2} / \sigma_{l}^{2}$, where $\mu_{l}$ and $\sigma_{l}^{2}$ denote the $l$ th element of $\boldsymbol{\mu}_{h}$ and the $l$ th main diagonal element of $\boldsymbol{C}_{h h}$, respectively. For convenience we apply the normalization $M_{\boldsymbol{h}}(1)=L$, and we note that for Rayleigh and Ricean fading $\boldsymbol{\mu}_{h}=\mathbf{0}_{L}$ and $\boldsymbol{\mu}_{h} \neq \mathbf{0}_{L}$, respectively.

The noise vector $\boldsymbol{n}$ is independent of $\boldsymbol{h}$ and normalized to $M_{\boldsymbol{n}}(1)=L$. We note that the elements of $\boldsymbol{n}$ may be statistically dependent, non-circularly symmetric, and non-Gaussian. The only condition that we impose on $\boldsymbol{n}$ is that all joint moments of the elements of $\boldsymbol{n}$ exist, i.e., $\mathcal{E}\left\{n_{1}^{\kappa_{1}}\left(n_{1}^{*}\right)^{\nu_{1}} n_{2}^{\kappa_{2}}\left(n_{2}^{*}\right)^{\nu_{2}} \cdots n_{L}^{\kappa_{L}}\left(n_{L}^{*}\right)^{\nu_{L}}\right\}<\infty$ for all $\kappa_{l} \geq 0, \nu_{l} \geq 0,1 \leq l \leq L$.

\footnotetext{
${ }^{2}$ The combining gain is also often referred to as "coding gain" in the literature, e.g. [4]. We prefer the term "combining gain" as channel coding is not applied here.

${ }^{3}$ We will extend our signal model in Section 3.2.3 to binary frequency-shift keying (BFSK) modulation.
} 


\section{Asymptotic Performance Analysis}

In this section, we develop asymptotic expressions for the BEPs and SEPs of coherent MRC, differential EGC, and NC for various modulation schemes. However, first we derive a general asymptotic result for the pairwise error probability (PEP) of quadratic diversity combining receivers.

\subsection{Asymptotic Pairwise Error Probability (PEP)}

For quadratic diversity combining schemes the PEP can be generally expressed as

$$
P_{e}(d)=\operatorname{Pr}\left\{\left\|\sqrt{\bar{\gamma}} \boldsymbol{h} e+\boldsymbol{n}_{1}\right\|^{2}<\left\|\boldsymbol{n}_{2}\right\|^{2}\right\},
$$

where $\boldsymbol{n}_{1}$ and $\boldsymbol{n}_{2}$ denote two noise vectors and $e$ is a complex scalar with $d^{2} \triangleq|e|^{2}$. $\boldsymbol{n}_{1}, \boldsymbol{n}_{2}$, and $e$ will be specified for different combining schemes in Section 3.2. Based on Eq. (4) we can express the conditional PEP as

$$
P_{e}\left(d \mid \boldsymbol{n}_{1}, \boldsymbol{n}_{2}\right)=\int_{0}^{\left\|\boldsymbol{n}_{2}\right\|^{2}} p_{\Delta}(x) \mathrm{d} x,
$$

where $p_{\Delta}(x)$ denotes the pdf of $\Delta \triangleq\|\boldsymbol{u}\|^{2}$ with $\boldsymbol{u} \triangleq \sqrt{\bar{\gamma}} \boldsymbol{h} e+\boldsymbol{n}_{1}$. Conditioned on $\boldsymbol{n}_{1}, \boldsymbol{u}$ is a Gaussian random vector with mean $\boldsymbol{\mu}_{u} \triangleq \mathcal{E}\left\{\boldsymbol{u} \mid \boldsymbol{n}_{1}\right\}=\sqrt{\bar{\gamma}} e \boldsymbol{\mu}_{h}+\boldsymbol{n}_{1}$ and covariance matrix $\boldsymbol{C}_{u u} \triangleq \mathcal{E}\left\{\boldsymbol{u} \boldsymbol{u}^{H} \mid \boldsymbol{n}_{1}\right\}=\bar{\gamma}|e|^{2} \boldsymbol{C}_{h h}$. Therefore, the Laplace transform $\Phi_{\Delta}(s)$ of $p_{\Delta}(x)$ can be expressed as [12]

$$
\Phi_{\Delta}(s) \triangleq \mathcal{E}\left\{e^{-s \Delta}\right\}=\frac{\exp \left(-s\left[\sqrt{\bar{\gamma}} e \boldsymbol{\mu}_{h}+\boldsymbol{n}_{1}\right]^{H}\left(\boldsymbol{I}_{L}+s \bar{\gamma}|e|^{2} \boldsymbol{C}_{h h}\right)^{-1}\left[\sqrt{\bar{\gamma}} e \boldsymbol{\mu}_{h}+\boldsymbol{n}_{1}\right]\right)}{\operatorname{det}\left(\boldsymbol{I}_{L}+s \bar{\gamma}|e|^{2} \boldsymbol{C}_{h h}\right)} .
$$

Eq. (6) reveals that for full rank fading correlation matrices $\boldsymbol{C}_{h h}$ and $\bar{\gamma} \rightarrow \infty$ the Laplace transform $\Phi_{\Delta}(s)$ can be simplified to

$$
\Phi_{\Delta}(s) \doteq \frac{\exp \left(-\left[\boldsymbol{\mu}_{h}+\boldsymbol{n}_{1} /(e \sqrt{\bar{\gamma}})\right]^{H} \boldsymbol{C}_{h h}^{-1}\left[\boldsymbol{\mu}_{h}+\boldsymbol{n}_{1} /(e \sqrt{\bar{\gamma}})\right]\right)}{\operatorname{det}\left(\boldsymbol{C}_{h h}\right) d^{2 L} \bar{\gamma}^{L}} s^{-L} .
$$

An asymptotic expression for $p_{\Delta}(x)$ can now be easily obtained by applying the inverse Laplace transform to Eq. (7). This result can then be used in Eq. (5) to obtain the asymptotic conditional PEP

$$
P_{e}\left(d \mid \boldsymbol{n}_{1}, \boldsymbol{n}_{2}\right) \doteq \frac{\exp \left(-\left[\boldsymbol{\mu}_{h}+\boldsymbol{n}_{1} /(e \sqrt{\bar{\gamma}})\right]^{H} \boldsymbol{C}_{h h}^{-1}\left[\boldsymbol{\mu}_{h}+\boldsymbol{n}_{1} /(e \sqrt{\bar{\gamma}})\right]\right)}{L ! \operatorname{det}\left(\boldsymbol{C}_{h h}\right) d^{2 L} \bar{\gamma}^{L}}\left\|\boldsymbol{n}_{2}\right\|^{2 L}
$$


Using the expansion $\exp (x)=\sum_{k=0}^{\infty} x^{k} / k$ !, we can rewrite the exponential function in Eq. (8) as

$$
\exp \left(-\left[\boldsymbol{\mu}_{h}+\boldsymbol{n}_{1} /(e \sqrt{\bar{\gamma}})\right]^{H} \boldsymbol{C}_{h h}^{-1}\left[\boldsymbol{\mu}_{h}+\boldsymbol{n}_{1} /(e \sqrt{\bar{\gamma}})\right]\right)=\exp \left(-\boldsymbol{\mu}_{h}^{H} \boldsymbol{C}_{h h}^{-1} \boldsymbol{\mu}_{h}\right)\left(1+f\left(\boldsymbol{n}_{1}\right) / \bar{\gamma}\right)
$$

where $f\left(\boldsymbol{n}_{1}\right)$ is implicitly defined in Eq. (9). Furthermore, $f\left(\boldsymbol{n}_{1}\right)$ can be written as a sum of products of the form $C_{\kappa_{1}, \nu_{1}, \cdots, \kappa_{L}, \nu_{L}} n_{1,1}^{\kappa_{1}}\left(n_{1,1}^{*}\right)^{\nu_{1}} n_{1,2}^{\kappa_{2}}\left(n_{1,2}^{*}\right)^{\nu_{2}} \cdots n_{1, L}^{\kappa_{L}}\left(n_{1, L}^{*}\right)^{\nu_{L}}$, where $C_{\kappa_{1}, \nu_{1}, \cdots, \kappa_{L}, \nu_{L}}$ are coefficients that are non-increasing in $\bar{\gamma}, n_{1, l}, 1 \leq l \leq L$, denotes the elements of $\boldsymbol{n}_{1}$, and $\kappa_{l} \geq 0$ and $\nu_{l} \geq 0$ are integers. Assuming now that all individual and joint moments of $\boldsymbol{n}_{1}$ and $\boldsymbol{n}_{2}$ exist (i.e., $\mathcal{E}\left\{n_{1,1}^{\kappa_{1,1}}\left(n_{1,1}^{*}\right)^{\nu_{1,1}} n_{2,1}^{\kappa_{2,1}}\left(n_{2,1}^{*}\right)^{\nu_{2,1}} \cdots n_{1, L}^{\kappa_{1, L}}\left(n_{1, L}^{*}\right)^{\nu_{1, L}} n_{2, L}^{\kappa_{2, L}}\left(n_{2, L}^{*}\right)^{\nu_{2, L}}\right\}<\infty$, where $n_{2, l}$ are the elements of

$\boldsymbol{n}_{2}$, and $\nu_{1, l} \geq 0, \nu_{2, l} \geq 0, \kappa_{1, l} \geq 0$, and $\left.\kappa_{2, l} \geq 0,1 \leq l \leq L\right)$, we obtain for $\bar{\gamma} \rightarrow \infty$ from Eqs. (8) and (9) for the asymptotic (unconditional) PEP the simple expression

$$
P_{e}(d)=\mathcal{E}\left\{P_{e}\left(d \mid \boldsymbol{n}_{1}, \boldsymbol{n}_{2}\right)\right\} \doteq \frac{p_{h} M_{\boldsymbol{n}_{2}}(L)}{L ! d^{2 L}} \bar{\gamma}^{-L}
$$

with

$$
p_{h} \triangleq \frac{\exp \left(-\boldsymbol{\mu}_{h}^{H} \boldsymbol{C}_{h h}^{-1} \boldsymbol{\mu}_{h}\right)}{\operatorname{det}\left(\boldsymbol{C}_{h h}\right)} .
$$

From Eq. (10) we observe that $\boldsymbol{n}_{1}$ has no influence on the asymptotic PEP. Furthermore, $\boldsymbol{n}_{2}$ affects the PEP via $M_{\boldsymbol{n}_{2}}(L)$, i.e., only the number of diversity branches $L$ determines which moment of $\left\|\boldsymbol{n}_{2}\right\|^{2}$ is relevant for the PEP but the mean $\boldsymbol{\mu}_{h}$ and the correlation matrix $\boldsymbol{C}_{h h}$ of $\boldsymbol{h}$ have no influence in this regard.

\subsection{Quadratic Diversity Combining Schemes}

In this section, we apply the general asymptotic PEP result in Eq. (10) to calculate the asymptotic SEP and BEP of coherent MRC, differential EGC, and NC. We note that diversity combining rules optimized for AWGN are in general suboptimum for non-Gaussian noise, of course. However, in practice, it may be unrealistic to assume that the receiver can accurately estimate the noise and interference statistics which also may change with time. Therefore, as a pragmatic and popular choice, we will adopt here quadratic combining schemes optimized for AWGN also for non-Gaussian noise. 


\subsubsection{Coherent Maximum Ratio Combining (MRC)}

The MRC decision rule can be expressed as $\hat{b}=\underset{b \in \mathcal{A}}{\operatorname{argmin}}\left\{\|\boldsymbol{r}-\sqrt{\bar{\gamma}} \boldsymbol{h} \tilde{b}\|^{2}\right\}$, where $\hat{b}$ and $\tilde{b}$ denote the estimated symbol and a hypothetical symbol, respectively. As a consequence, Eq. (4) is valid for MRC if we let $\boldsymbol{n}_{1}=\boldsymbol{n}_{2}=\boldsymbol{n}$ and $e \triangleq b-\bar{b}$, where $b, \bar{b} \in \mathcal{A}$ and $b \neq \bar{b}$. For high SNR the SEP will be dominated by the PEP of the nearest-neighbor signal points of $\mathcal{A}$. Therefore, exploiting Eq. (10) we obtain for the asymptotic SEP

$$
\mathrm{SEP} \doteq \beta_{M} P_{e}\left(d_{M}\right) \doteq \frac{\beta_{M} p_{h} M_{\boldsymbol{n}}(L)}{L ! d_{M}^{2 L}} \bar{\gamma}^{-L},
$$

where $d_{M}$ denotes the minimum Euclidean distance of $\mathcal{A}$ and $\beta_{M}$ is the average number of minimumdistance neighbors. For convenience, the values of $\beta_{M}$ and $d_{M}$ are listed in Table 1 for commonly used constellations $\mathcal{A}$ such as $M$-ary pulse amplitude modulation ( $M$-PAM), $M$-ary quadrature amplitude modulation ( $M-\mathrm{QAM})$, and $M$-ary phase-shift keying ( $M-\mathrm{PSK})$.

\subsubsection{Differential Equal Gain (EGC) Combining}

Differential $\mathrm{EGC}^{4}$ is applicable to differential $M$-PSK transmission. In differential $M$-PSK the transmitted $M$-PSK symbols are obtained as $b[k]=a[k] b[k-1]$, where the differential symbols $a[k]$ also belong to an $M$-PSK constellation $\mathcal{A}$. The differential EGC decision rule can be expressed as $\hat{a}[k]=\underset{\tilde{a}[k] \in \mathcal{A}}{\operatorname{argmin}}\left\{\|\boldsymbol{r}[k]-\tilde{a}[k] \boldsymbol{r}[k-1]\|^{2}\right\}$, where $\hat{a}[k]$ and $\tilde{a}[k]$ are the estimated symbol and a hypothetical symbol, respectively. Therefore, Eq. (4) is applicable to differential EGC if we let $e \triangleq(a[k]-\bar{a}[k]) b[k-1]$, where $a[k], \bar{a}[k] \in \mathcal{A}$ and $a[k] \neq \bar{a}[k], \boldsymbol{n}_{1} \triangleq \boldsymbol{n}[k]-\bar{a}[k] \boldsymbol{n}[k-1]$, and

$$
\boldsymbol{n}_{2}=\tilde{\boldsymbol{n}} \triangleq \boldsymbol{n}[k]-a[k] \boldsymbol{n}[k-1]
$$

If the marginal pdfs $p_{n_{l}}\left(n_{l}\right), 1 \leq l \leq L$, of all components of $\boldsymbol{n}[k-1]$ are circular, i.e., $p_{n_{l}}\left(n_{l}\right)=$ $p_{n_{l}}\left(e^{j \varphi} n_{l}\right)$ for all $\varphi \in[-\pi, \pi)[13]$, and $\boldsymbol{n}[k]$ and $\boldsymbol{n}[k-1]$ are statistically independent, $a[k]$ has no influence on the distribution of $\tilde{\boldsymbol{n}}$ and $\tilde{\boldsymbol{n}} \triangleq \boldsymbol{n}[k]-\boldsymbol{n}[k-1]$ may be used instead of the definition in Eq. (13). Recalling again that the asymptotic SEP is dominated by the PEPs of the nearest-neighbor

\footnotetext{
${ }^{4}$ Differential EGC is also referred to as "differentially coherent" EGC and "post-detection" EGC in the literature, cf. e.g. [1].
} 
signal points of $\mathcal{A}$, we obtain with Eq. (10) for differential EGC the expression

$$
\mathrm{SEP} \doteq \frac{\beta_{M} p_{h} M_{\tilde{\boldsymbol{n}}}(L)}{L ! d_{M}^{2 L}} \bar{\gamma}^{-L},
$$

with $\beta_{M}$ and $d_{M}$ as specified in Table 1 for $M$-PSK.

\subsubsection{Noncoherent Combining (NC)}

In this subsection, we consider NC (also referred to as "square law combining") of BFSK. For BFSK the channel model in Section 2.2 has to be slightly modified since there are now two matched filters per receive antenna. We collect the outputs of these two matched filters in vectors $r$ and $\bar{r}$ which can be modeled as $\boldsymbol{r} \triangleq \sqrt{\bar{\gamma}} \boldsymbol{h} b+\boldsymbol{n}$ and $\overline{\boldsymbol{r}} \triangleq \sqrt{\bar{\gamma}} \boldsymbol{h} \bar{b}+\overline{\boldsymbol{n}}$, where $b, \bar{b} \in\{0,1\}$ and $b \neq \bar{b}$ [2]. We note that $\boldsymbol{n}$ and $\overline{\boldsymbol{n}}$ are identically distributed and statistically independent if the channel noise is Gaussian [2]. For non-Gaussian noise $\boldsymbol{n}$ and $\bar{n}$ are still identically distributed but not necessarily statistically independent. Fortunately, the statistical dependence of $\boldsymbol{n}$ and $\bar{n}$ does not affect the applicability of the results of Section 3.1. For NC the magnitudes of $\|\boldsymbol{r}\|^{2}$ and $\|\overline{\boldsymbol{r}}\|^{2}$ are compared to arrive at a decision on the transmitted symbol and Eq. (4) is obviously applicable with $e=1$. Therefore, the asymptotic SEP of BFSK can be expressed as

$$
\mathrm{SEP} \doteq \frac{p_{h} M_{\boldsymbol{n}}(L)}{L ! d_{2}^{2 L}} \bar{\gamma}^{-L},
$$

where $d_{2}=|e|=1$. We note that unlike for linear modulations, $d_{2}$ is not the Euclidean distance between the signal points of the BFSK constellation in the signal space.

\subsubsection{Bit Error Probability (BEP)}

For non-binary modulation with Gray mapping, the asymptotic BEPs can be obtained from the corresponding SEPs as [2]

$$
\mathrm{BEP} \doteq \frac{\mathrm{SEP}}{\log _{2}(M)} .
$$

Using Eqs. (12), (14)-(16) the SEPs and BEPs of MRC, differential EGC, and NC can be easily calculated as long as closed-form expressions for the moments $M_{\boldsymbol{n}}(L)$ and $M_{\tilde{\boldsymbol{n}}}(L)$ are available. The calculation of these moments for practically relevant types of noise and interference is addressed in Section 4. 


\subsection{Combining Gain and Comparison}

A comparison of Eqs. (12), (14), and (15) with Eq. (1) shows immediately that the diversity gain of all considered quadratic combining schemes is $G_{d}=L$ independent of the type of noise. Furthermore, on a logarithmic scale, the combining gain can be expressed in a unified manner as

$$
G_{c}=\frac{10}{L} \log _{10}(L !)+\frac{10}{L} \log _{10}\left(\frac{d_{M}^{2 L}}{\beta_{M}}\right)-\frac{10}{L} \log _{10}\left(p_{h}\right)-\frac{10}{L} \log _{10}\left(M_{\boldsymbol{n}_{2}}(L)\right),
$$

where $\boldsymbol{n}_{2}=\boldsymbol{n}$ and $\boldsymbol{n}_{2}=\tilde{\boldsymbol{n}}$ for MRC, NC and differential EGC, respectively. The second, the third, and the fourth term of Eq. (17) show the dependence of $G_{c}$ on the modulation scheme, the channel statistics, and the noise statistics, respectively. Eq. (17) reveals that for a given $L$ the modulation scheme, the channel statistics (i.e., $\boldsymbol{\mu}_{h}$ and $\boldsymbol{C}_{h h}$ ), and the noise statistics independently contribute to the combining gain. This new result is somewhat unexpected and means that increasing the correlation of the fading or changing the modulation scheme will horizontally shift the asymptotic SEP curves (on a log-log scale) for different types of noise by the same amount. On the other hand, the asymptotic performance difference of different types of noise is independent of the modulation scheme and the channel statistics.

In the following, we will consider the combining gain for three special cases more in detail.

1) $L=1$ : Since $M_{\boldsymbol{n}}(1)=L$ is valid for all types of noise, Eq. (15) shows that for $L=1$ the asymptotic error rate performance of MRC and NC is independent of the type of noise as long as all joint moments of $\boldsymbol{n}$ are finite. The same is true for EGC since in this case $M_{\tilde{\boldsymbol{n}}}(1)=$ $\mathcal{E}\{\mid n[k]-a[k] n[k-1]\}=2 M_{\boldsymbol{n}}(1)-\Re\left\{\mathcal{E}\left\{a^{*}[k]\right\} \mathcal{E}\left\{n[k] n^{*}[k-1]\right\}\right\}=2 L$ holds for all types of noise because $\mathcal{E}\left\{a^{*}[k]\right\}=0$ for $M$-PSK. For $L>1$ both $M_{\boldsymbol{n}}(L)$ and $M_{\tilde{\boldsymbol{n}}}(L)$ depend on the type of noise and the same is true for the asymptotic error rate performance, of course.

2) $M R C$ vs. NC: It is interesting to compare MRC and NC for binary modulation. Using $\beta_{2}=1$ and the appropriate values for $d_{2}$ in Eq. (17) shows that for any given number of diversity branches $L$ the asymptotic SNR loss of BFSK with NC compared to BPSK with coherent MRC is $6 \mathrm{~dB}$. While this is a well known result for channels impaired by i.i.d. AWGN [2], our derivation shows that the same loss results for any type of noise and interference as long as all of its moments exist.

3) $M R C$ vs. differential EGC: Eq. (17) also reveals that for $M$-PSK the relative asymptotic 
performance loss of differential EGC compared to coherent MRC is

$$
\Delta G_{E M}=\frac{10}{L} \log _{10}\left(\frac{M_{\tilde{\boldsymbol{n}}}(L)}{M_{\boldsymbol{n}}(L)}\right) .
$$

Eq. (18) shows that $\Delta G_{E M}$ only depends on the number of diversity branches $L$ and the noise statistics. For $L=1$ we obtain $\Delta G_{E M}=3 \mathrm{~dB}$ for all types of noise $\boldsymbol{n}$ with finite joint moments. Similarly, it will be shown in Section 4, that for i.i.d. AWGN $\Delta G_{E M}=3 \mathrm{~dB}$ holds for all $L$. However, for non-Gaussian noise and $L>1$ the performance loss $\Delta G_{E M}$ depends on $L$ and may be smaller or larger than $3 \mathrm{~dB}$, cf. Section 4.

\section{Calculation of Noise Moments}

The main difficulty in evaluating the asymptotic SEP expressions in Eqs. (12), (14), and (15) is the calculation of the moments $M_{\boldsymbol{n}}(L)$ and $M_{\tilde{\boldsymbol{n}}}(L)$, respectively. In this section, we derive the moments of some basic types of noise and we introduce general rules that facilitate the calculation of the moments of more complicated, composite noises. For convenience we discuss spatially independent and spatially dependent noise separately. We also present a Monte-Carlo based approach to moment calculation for cases where closed-form results cannot be obtained.

\subsection{Spatially Independent Noise}

For many practically relevant scenarios the noises in different diversity branches are mutually independent. In this case, the multinomial expansion [14] can be used to simplify $M_{\boldsymbol{n}}(L)$ to

$$
M_{n}(L)=\sum_{k_{1}+\ldots+k_{L}=L}\left(\begin{array}{c}
L \\
k_{1}, \ldots, k_{L}
\end{array}\right) M_{n_{1}}\left(k_{1}\right) \cdot \ldots \cdot M_{n_{L}}\left(k_{L}\right) .
$$

Therefore, for independent noise the calculation of $M_{\boldsymbol{n}}(L)$ reduces to finding the $L(L+1)$ scalar moments $M_{n_{l}}\left(k_{l}\right), 0 \leq k_{l} \leq L, 1 \leq l \leq L$. This motivates us to consider the moments of scalar $\mathrm{RV}$ s more in detail in the following subsections.

\subsubsection{Moments of Elementary Scalar RVs}

In Table 2, we provide the moments $M_{n}(N)$ of elementary scalar RVs which are frequently encountered in the context of noise and interference. In particular, we consider Gaussian RVs with mean $\mu_{n}$ 
and variance $\sigma_{n}^{2}$, Gaussian mixture RVs, interference with a fixed channel, and M-PSK interference with a random channel phase. The non-Gaussian RVs are briefly discussed in the following.

E1) Gaussian mixture RVs: Gaussian mixture RVs are used to model the combined effect of Gaussian background noise and man-made, impulsive noise [9]. The pdf of Gaussian mixture noise with $I$ terms is given by

$$
p_{n}(n)=\sum_{k=1}^{I} \frac{c_{k}}{\pi \sigma_{k}^{2}} \exp \left(-\frac{|n|^{2}}{\sigma_{k}^{2}}\right),
$$

where $c_{k}>0, \sum_{k=1}^{I} c_{k}=1$, and $\sigma_{k}^{2}, 1 \leq k \leq I$, are constants. Special cases of Gaussian mixture noise include Middelton's Class-A noise $(I \rightarrow \infty)[15]$ and $\epsilon$-mixture noise $(I=2) . \epsilon$-mixture noise with variance $\sigma_{n}^{2}$ is characterized by $c_{1}=1-\epsilon, c_{2}=\epsilon, \sigma_{1}^{2}=\sigma_{n}^{2} /(1-\epsilon+\kappa \epsilon), \sigma_{2}^{2}=\kappa \sigma_{n}^{2} /(1-\epsilon+\kappa \epsilon)$, $0 \leq \epsilon<1$, and $\kappa>1$.

E2) Interference with fixed channel: Interference with a fixed channel can be modeled as

$$
n=\sum_{k=k_{l}}^{k_{u}} g[k] i[k]
$$

where $k_{l}, k_{u}, g[k]$, and $i[k]$ denote the lower limit, the upper limit, fixed (in general complex) coefficients, and i.i.d. interference symbols taken from an $M_{i}$-ary symbol alphabet. Eq. (21) can be used to model multiple synchronous, a single asynchronous, or multiple asynchronous co-channel interferers. For example, for $I$ synchronous interferers we have $k_{l}=1, k_{u}=I$, and $g[k]$ and $i[k]$ denote the gain and the transmitted symbol of the $k$ th interferer. In contrast, for a single asynchronous interferer $i[k]$ denotes symbols transmitted by the interferer in symbol intervals $k_{l} \leq$ $k \leq k_{u}$ and $g[k] \triangleq g(k T+\tau)$, where $g(t), T$, and $\tau$ are the overall interference pulse shape, the symbol duration, and the delay of the interferer compared to the desired user, respectively. $k_{l}$ and $k_{u}$ are appropriately chosen to ensure $g(k T+\tau) \approx 0$ for $k<k_{l}$ and $k>k_{u}$, respectively. The generalized moments of $n$ are given in Table 2, where the set $\mathcal{S}$ includes the $M_{i}^{k_{u}-k_{l}+1}$ possible values of $n$ corresponding to all $M_{i}^{k_{u}-k_{l}+1}$ possible combinations of $i[k], k_{l} \leq k \leq k_{u}$, cf. Eq. (21).

E3) Synchronous $M_{i}-P S K$ interference with random channel phase (CP): In this case, the noise is given by $n=g e^{j \varphi} i[k]$, where $g, \varphi$, and $i[k]$ denote the channel gain, the random CP uniformly distributed in $[-\pi, \pi)$, and the interfering $M_{i}-$ PSK symbol, respectively. Note that $i[k]$ has no influence on $M_{n}(N)$ given in Table 2. 


\subsubsection{Calculus for Moments of Scalar RVs}

In practice, the noise may consist of sums or/and products of different RVs. For example, co-channel interference and Gaussian background noise may impair the received signal at the same time. For the case where the involved RVs are statistically independent, we establish some general combining rules for their scalar moments. The derivation of most of these combining rules (CRs) is relatively straightforward and, due to space limitation, we do not provide any proofs here. In the following, we assume that $n_{1}$ and $n_{2}$ are statistically independent RVs.

CR1) Product $n=n_{1} n_{2}$ : It is easy to show that the $N$ th moment of $|n|^{2}$ is given by

$$
M_{n}(N)=\mathcal{E}\left\{\left|n_{1} n_{2}\right|^{2 N}\right\}=\mathcal{E}\left\{\left|n_{1}\right|^{2 N}\right\} \mathcal{E}\left\{\left|n_{2}\right|^{2 N}\right\}=M_{n_{1}}(N) M_{n_{2}}(N) .
$$

CR2) Sum $n=n_{1}+n_{2}$ : To arrive at a simple result, we have to assume that the pdfs of $n_{1}$ and $n_{2}$ are both circular [13]. For example, zero-mean Gaussian RVs and the RVs in E1) and E3) are circular, whereas non-zero mean Gaussian RVs and the RV in E2) are not circular. Assuming circularity, we obtain after some straightforward manipulations

$$
M_{n}(N)=\sum_{k_{1}+2 k_{2}+k_{3}=N} \frac{N !}{k_{1} !\left(k_{2} !\right)^{2} k_{3} !} M_{n_{1}}\left(k_{1}+k_{2}\right) M_{n_{2}}\left(k_{2}+k_{3}\right) .
$$

For example, for $L=1,2$, and 3 Eq. (23) yields $M_{n}(1)=M_{n_{1}}(1)+M_{n_{2}}(1), M_{n}(2)=M_{n_{1}}(2)+$ $4 M_{n_{1}}(1) M_{n_{2}}(1)+M_{n_{2}}(2)$, and $M_{n}(3)=M_{n_{1}}(3)+9 M_{n_{1}}(1) M_{n_{2}}(2)+9 M_{n_{1}}(2) M_{n_{2}}(1)+M_{n_{2}}(3)$.

CR3) Scaling $n=\xi n_{1}, \xi$ constant: The $N$ th moment of $|n|^{2}$ can be obtained as

$$
M_{n}(N)=|\xi|^{2 N} M_{n_{1}}(N)
$$

Based on the scalar moments of the elementary RVs given in Table 2 and the combining rules established in this section, the moments of a large class of composite noises can be calculated.

\subsubsection{Moments of Composite Scalar RVs}

In order to illustrate the application of the moment combining rules (R1)-CR5) established in the previous subsection, we briefly discuss two relevant examples.

E4) Differential EGC with i.i.d. noise: Assuming circular noise, the SEP of differential EGC depends on the moments $M_{\tilde{\boldsymbol{n}}}(L)$ of $\tilde{\boldsymbol{n}}=\boldsymbol{n}[k]-\boldsymbol{n}[k-1]$, cf. Eqs. (13), (14). Therefore, if the noise 
$n_{l}[k], 1 \leq l \leq L$, is spatially and temporally statistically independent, $M_{\tilde{\boldsymbol{n}}}(L)$ can be calculated from the scalar moments by applying Eqs. (19) and (23). Examples where the noise is spatially and temporally statistically independent include the conventional AWGN model and Gaussian mixture noise following "Model II" in [9]. Spatially independent Gaussian mixture noise is an appropriate model for impulsive noise if the phenomenon causing the impulsive behavior affects the antennas independently, see [9] for a detailed discussion.

We are now also in a position to shed some more light onto the performance loss of differential EGC compared to coherent MRC. For example, assuming spatially and temporally i.i.d. noise (i.e., $\left.M_{n_{l}[k]}(N)=M_{n}(N), 1 \leq l \leq L, \forall k\right)$ and $L=1$ Eq. (19) yields $M_{n}(1)=M_{n}(1)$. Since a similar relation holds for $M_{\tilde{n}}(1)$, we obtain $\Delta G_{E M}=10 \log _{10}\left(M_{\tilde{n}}(1) / M_{n}(1)\right)$. On the other hand, according to Eq. (23) $M_{\tilde{n}}(1)=2 M_{n}(1)$. Therefore, we find that for $L=1$ and circular i.i.d. (possibly non-Gaussian) noise, differential EGC suffers from a loss of $\Delta G_{E M}=3 \mathrm{~dB}$ compared to coherent MRC. For $L>1$ the loss $\Delta G_{E M}$ may be different from $3 \mathrm{~dB}$ and depends on the type of noise. For example, for $L=2$ we obtain

$$
\Delta G_{E M}=5 \log _{10}\left(2 \frac{M_{n}(2)+4 M_{n}^{2}(1)}{M_{n}(2)+M_{n}^{2}(1)}\right),
$$

which can be simplified to

$$
\Delta G_{E M}=3 \mathrm{~dB}+5 \log _{10}\left(\frac{\left(1-\epsilon+\epsilon \kappa^{2}\right)+2(1-\epsilon+\epsilon \kappa)^{2}}{2\left(1-\epsilon+\epsilon \kappa^{2}\right)+(1-\epsilon+\epsilon \kappa)^{2}}\right)
$$

for i.i.d. $\epsilon$-mixture noise. For $\epsilon=0$ the $\epsilon$-mixture noise degenerates to Gaussian noise and $\Delta G_{E M}=$ $3 \mathrm{~dB}$ follows from Eq. (26). On the other hand, for impulsive noise (i.e., $\epsilon>0) \Delta G_{E M} \neq 3 \mathrm{~dB}$ holds in general.

E5) Ricean faded $M_{i}-P S K$ interferer: The interference caused by a synchronous Ricean faded $M_{i}$-PSK co-channel interferer $i[k]$ can be modeled as $n_{l}=\left(e^{j \varphi_{l}} g_{l}+n_{l}^{\prime}\right) i[k], 1 \leq l \leq L$, where $e^{j \varphi_{l}} g_{l}$ and $n_{l}^{\prime}$ denote the direct and the specular Ricean components, respectively. Assuming that $\varphi_{l}$ is uniformly distributed in $[-\pi, \pi), e^{j \varphi_{l}} g_{l} i[k]$ can be modeled by Example E3), and $n_{l}^{\prime} i[k]$ is a zero mean Gaussian RV. Since the $M_{i}$-PSK symbol $i[k]$ does not affect the distributions of $e^{j \varphi_{l}} g_{l} i[k]$ and $n_{l}^{\prime} i[k]$, we can drop it in the following. Furthermore, since both $e^{j \varphi_{l}} g_{l}$ and $n_{l}^{\prime}$ are circular we can calculate the moments $M_{n_{l}}(N)$ using Eq. (23) and Table 2. Once these scalar moments are calculated, the moments of $M_{\boldsymbol{n}}(L)$ can be obtained from Eq. (19). 


\subsubsection{I.I.D. Additive White Gaussian Noise (AWGN)}

Since the case of (i.i.d.) AWGN has been extensively studied in the literature, it is instructive to verify our novel asymptotic SEP results for this special case. Here, $\|\boldsymbol{n}\|^{2}$ and $\|\tilde{\boldsymbol{n}}\|^{2}$ are both chisquare distributed RVs with $2 L$ degrees of freedom and it is straightforward to derive $M_{n}(L)$ as given in Table 3 and

$$
M_{\tilde{\boldsymbol{n}}}(L)=2^{L} M_{\boldsymbol{n}}(L) .
$$

Therefore, according to Eq. (18), for AWGN the asymptotic performance loss $\Delta G_{E M}$ of differential EGC compared to coherent MRC is $3 \mathrm{~dB}$ independent of the number of diversity branches and independent of the mean and the covariance matrix of $\boldsymbol{h}$. For the special case of BPSK transmission over i.i.d. Rayleigh fading channels this $3 \mathrm{~dB}$ loss is a well-known result, cf. [2, Section 14.4.1].

For coherent MRC our results for the special case of AWGN can be compared with those given in [4]. On the other hand, an asymptotic analysis of differential EGC for general Ricean fading does not seem to be available in the literature even for AWGN. For NC the case of independent Ricean fading and AWGN was considered in [3], but our asymptotic results for correlated Rayleigh and Ricean fading seem to be new even for AWGN. For i.i.d. Rayleigh fading and AWGN it can be shown that Eq. (12) for BPSK with MRC, Eq. (14) for BPSK with differential EGC, and Eq. (15) for BFSK with NC are equivalent to [2, Eq. (14.4-18)], [2, Eq. (14.4-28)], and [2, Eq. (14.4-33)], respectively.

\subsection{Spatially Dependent Noise}

In this subsection, we illustrate the calculation of the moments $M_{\boldsymbol{n}}(L)$ and $M_{\tilde{n}}(L)$ for spatially dependent noise for three different practically important types of noise and interference.

E6) Correlated Gaussian noise/interference: We consider the case where $\boldsymbol{n}$ can be modeled as a zero-mean correlated Gaussian RVV with covariance matrix $\boldsymbol{C}_{n n} \triangleq \mathcal{E}\left\{\boldsymbol{n} \boldsymbol{n}^{H}\right\}$. Such a model applies for example if the received vector is impaired by $I$ Rayleigh faded synchronous $M_{i}-\mathrm{PSK}$ co-channel interferers $i[k][16,17]$ and AWGN

$$
\boldsymbol{n}=\sum_{k=1}^{I} \boldsymbol{g}[k] i[k]+\boldsymbol{n}_{0}
$$


where $\boldsymbol{g}[k]$ are mutually independent complex zero-mean Gaussian RVVs with covariance matrices $\boldsymbol{C}_{g[k] g[k]} \triangleq \mathcal{E}\left\{\boldsymbol{g}[k] \boldsymbol{g}^{H}[k]\right\}, 1 \leq k \leq I$, and $\boldsymbol{n}_{0}$ is the AWGN vector. Because of the mutual independence of the fading vectors and the constant envelope of the $M_{i}$-PSK interference signals $(|i[k]|=1,1 \leq k \leq I), \boldsymbol{n}$ is a Gaussian vector with covariance matrix $\boldsymbol{C}_{n n}=\sum_{k=1}^{I} \boldsymbol{C}_{g[k] g[k]}+$ $\sigma_{n_{0}}^{2} \boldsymbol{I}_{L}$, where $\sigma_{n_{0}}^{2}$ denotes the variance of the elements of $\boldsymbol{n}_{0}$. As shown in Table 3, $M_{\boldsymbol{n}}(L)$ can be efficiently expressed in terms of the $L$ eigenvalues $\lambda_{k}, 1 \leq k \leq L$, of $\boldsymbol{C}_{n n}$. For the special case $L=2, M_{\boldsymbol{n}}(L)$ can be simplified to

$$
M_{\boldsymbol{n}}(2)=2\left(4-\operatorname{det}\left(\boldsymbol{C}_{n n}\right)\right)
$$

where we have used the normalization $M_{\boldsymbol{n}}(1)=L$. Interestingly, the moment assumes its minimum value $M_{\boldsymbol{n}}(L)=(2 L-1) ! /(L-1)$ ! for the special case of uncorrelated Gaussian noise $\left(\lambda_{k}=1\right.$, $1 \leq k \leq L)$ and its maximum value $M_{\boldsymbol{n}}(L)=L^{L} L$ ! for fully correlated noise $\left(\lambda_{1}=L\right.$ and $\lambda_{k}=0$, $2 \leq k \leq L)$. Therefore, using Eq. (12) we can bound the asymptotic SEP of MRC in correlated Gaussian noise as

$$
\left(\begin{array}{c}
2 L-1 \\
L
\end{array}\right) \frac{\beta_{M} p_{h}}{d_{M}^{2 L}} \bar{\gamma}^{-L} \leq \mathrm{SEP} \leq L^{L} \frac{\beta_{M} p_{h}}{d_{M}^{2 L}} \bar{\gamma}^{-L} .
$$

A similar result can be derived for NC, cf. Eq. (15). The combining gain loss $\Delta G_{c}$ caused in MRC and NC by fully correlated Gaussian noise compared to i.i.d. AWGN is $\Delta G_{c}(L)=10 \log _{10}(L[L !(L-$ $\left.1) ! /(2 L-1) !]^{1 / L}\right)$. For example, for $L=1,2$, and 3 we obtain $\Delta G_{c}(1)=0 \mathrm{~dB}, \Delta G_{c}(2)=0.6 \mathrm{~dB}$, and $\Delta G_{c}(3)=1.4 \mathrm{~dB}$, respectively. For large $L$, we can use Stirling's formula $\left(x ! \approx \sqrt{2 \pi} e^{-x} x^{x+1 / 2}\right.$, $x \gg 1)$ to obtain $\Delta G_{c}(L) \approx 10 \log _{10}(\sqrt[L]{2 \sqrt{L \pi}} L / 4) \approx 10 \log _{10}(L / 4), L \gg 1$, which shows that the performance loss due to noise correlation increases with $L$. It is interesting to note that not only correlation of the fading gains (elements of $\boldsymbol{h}$ ) has an adverse effect on the performance of MRC and NC but also correlation of the noise in different diversity branches.

E7) Asynchronous co-channel interference in correlated Rayleigh fading: A single Rayleigh faded asynchronous co-channel interferer can be modeled as

$$
\boldsymbol{n}=\boldsymbol{g} \cdot z,
$$

where $\boldsymbol{g}$ is a correlated zero-mean Gaussian RVV and $z$ can be modeled as in Example E2), i.e., $z=\sum_{k=k_{l}}^{k_{u}} g[k] i[k]$, cf. Eq. (21). Since $\boldsymbol{g}$ and $z$ are statistically independent, the moments of $\boldsymbol{n}$ can 
be calculated as

$$
M_{\boldsymbol{n}}(L) \triangleq \mathcal{E}\left\{\|\boldsymbol{g} z\|^{2 L}\right\}=\mathcal{E}\left\{\|\boldsymbol{g}\|^{2 L}\right\} \mathcal{E}\left\{|z|^{2 L}\right\}=M_{\boldsymbol{g}}(L) M_{z}(L)
$$

where $M_{z}(L)$ and $M_{\boldsymbol{g}}(L)$ can be obtained from Table 2 (interference with fixed channel) and Table 3 (correlated Gaussian RVV), respectively.

We observe from Eq. (32) that for asynchronous co-channel interference correlation of the interference channel has the same adverse effect as in case of Gaussian interference, cf. E6).

Assuming time-invariant fading the relevant noise term for differential EGC is given by $\tilde{\boldsymbol{n}}=$ $\boldsymbol{g}(n[k]-a[k] n[k-1])$, where $n[k]$ and $a[k] n[k-1]$ are the interference contributions in two successive symbol intervals. Therefore, $\tilde{n}=n[k]-a[k] n[k-1]$ can also be modeled as in Eq. (21) if $g[k]$ and $i[k]$ are replaced with appropriately defined effective coefficients $\tilde{g}[k]$ and effective interference symbol $\tilde{i}[k]$, respectively. In order to illustrate this we consider the special case of a single synchronous cochannel interferer, i.e., $\tilde{n}=i[k]-a[k] i[k-1]$. Assuming that the desired user and the interferer use the same $M-P S K$ constellation, i.e., $M_{i}=M$, we can simplify $\tilde{n}$ to $\tilde{n}=i[k]-i[k-1]$, which is in the form of Eq. (21). The moment of $\tilde{n}$ can be calculated to $M_{\tilde{n}}(L)=\frac{1}{M} 2^{L} \sum_{m=0}^{M-1}[1-\cos (2 \pi m / M)]^{L}$. Therefore, since $M_{n}(L)=1$ for $M$-PSK, the performance loss of differential EGC compared to MRC is given by

$$
\Delta G_{E M}=3 \mathrm{~dB}+\frac{10}{L} \log _{10}\left(\frac{1}{M} \sum_{m=0}^{M-1}\left[1-\cos \left(\frac{2 \pi}{M} m\right)\right]^{L}\right) .
$$

Eq. (33) reveals that the performance loss suffered by differential EGC in correlated co-channel interference depends only on $L$ and the adopted $M$-PSK constellation. For example, for BPSK $\Delta G_{E M}=3(2 L-1) / L \mathrm{~dB}$, whereas for 4-PSK $\Delta G_{E M}=3 \mathrm{~dB}+10 \log \left(2 \sqrt[L]{2^{-2}+2^{-(L+1)}}\right)$. For both BPSK and 4-PSK we obtain $\Delta G_{E M}=3 \mathrm{~dB}$ for $L=1$ and $\Delta G_{E M}=6 \mathrm{~dB}$ for $L \gg 1$. For $M \gg 1$ the sum in Eq. (33) can be approximated by an integral. This leads to

$$
\Delta G_{E M}=6 \mathrm{~dB}-10 \log _{10}\left(\sqrt[L]{\frac{(2 L) ! !}{(2 L-1) ! !}}\right),
$$

which yields $\Delta G_{E M}=3 \mathrm{~dB}, 3.9 \mathrm{~dB}$, and $4.3 \mathrm{~dB}$ for $L=1,2$, and 3, respectively, and approaches 6 $\mathrm{dB}$ as $L$ increases. From these considerations we conjecture that for a single synchronous $M-P S K$ co-channel interferer and any constellation size $M$ the asymptotic performance loss of differential EGC compared to MRC is between $3 \mathrm{~dB}$ and $6 \mathrm{~dB}$, where the higher value is approached as $L$ 
increases. Clearly, this is a very different behavior than that observed for AWGN, where we obtained $\Delta G_{c}=3 \mathrm{~dB}$ for all $L$, cf. Section 4.1.4.

We also note that since correlation increases $M_{\boldsymbol{g}}(L)$, cf. E6, Eqs. (32), (12), (14), and (15) show that, similar to the Gaussian interference case, correlation in the interference channel has an adverse effect on the performance also for asynchronous co-channel interference.

E8) Spatially dependent Gaussian mixture noise: The pdf of spatially dependent Gaussian mixture noise ("Model I" in [9]) is given by

$$
p_{\boldsymbol{n}}(\boldsymbol{n})=\sum_{k=1}^{I} \frac{c_{k}}{\pi^{L} \sigma_{k}^{2 L}} \exp \left(-\frac{\|\boldsymbol{n}\|^{2}}{\sigma_{k}^{2}}\right)
$$

where $c_{k}>0, \sum_{k=1}^{I} c_{k}=1$, and $\sigma_{k}^{2}, 1 \leq k \leq I$, are constants. Spatially dependent Gaussian mixture noise may be specialized to a multi-dimensional version of Middelton's Class-A noise $(I \rightarrow$ $\infty)$ and multi-dimensional $\epsilon$-mixture noise $(I=2)$. Spatially dependent Gaussian mixture noise is an appropriate model for impulsive noise if the physical process causing the impulsive behavior affects all antennas at the same time, see [9] for more discussion. The moments $M_{\boldsymbol{n}}(L)$ for spatially dependent Gaussian mixture noise are given in Table 3.

Assuming temporally independent, spatially dependent Gaussian mixture noise, it is easy to show that $\tilde{\boldsymbol{n}}$ is also a Gaussian mixture RVV whose pdf can be obtained from Eq. (35) by replacing $I$, $c_{k}$, and $\sigma_{k}^{2}$ by $\tilde{I} \triangleq I(I+1) / 2, \tilde{c}_{k}$, and $\tilde{\sigma}_{k}^{2}$, respectively. The latter two parameters are defined as $\tilde{c}_{k} \triangleq c_{k}^{2}$ and $\tilde{\sigma}_{k}^{2} \triangleq 2 \sigma_{k}^{2}$ for $1 \leq k \leq I$, and $\tilde{c}_{k} \triangleq 2 c_{i} c_{j}$ and $\tilde{\sigma}_{k}^{2} \triangleq \sigma_{i}^{2}+\sigma_{j}^{2}$ for $I+1 \leq k \leq \tilde{I}$, $1 \leq i \leq I, 1 \leq j \leq I, i \neq j$. With these definitions, the combining gain loss of differential EGC compared to coherent MRC can be expressed as

$$
\Delta G_{E M}=\frac{10}{L} \log _{10}\left(\frac{\sum_{k=1}^{\tilde{I}} \tilde{c}_{k} \tilde{\sigma}_{k}^{2 L}}{\sum_{k=1}^{I} c_{k} \sigma_{k}^{2 L}}\right)
$$

which for $\epsilon$-mixture noise can be simplified to

$$
\Delta G_{E M}=\frac{10}{L} \log _{10}\left(\frac{(1-\epsilon)^{2} 2^{L}+2 \epsilon(1-\epsilon)(\kappa+1)^{L}+\epsilon^{2}(2 \kappa)^{L}}{1-\epsilon+\epsilon \kappa^{L}}\right) .
$$

For $\epsilon=0$ (Gaussian case) Eq. (37) yields $\Delta G_{E M}=3 \mathrm{~dB}$ as expected. However, for $\epsilon>0$ and $\kappa>1$ differential EGC will cause a loss different from $3 \mathrm{~dB}$. For example, for $\epsilon=0.25$ and $\kappa=10$ we obtain from Eq. (37) $\Delta G_{E M}=3 \mathrm{~dB}, 2.25 \mathrm{~dB}$, and $2.0 \mathrm{~dB}$ for $L=1,2$, and 3, respectively. 


\subsection{Calculation by Monte-Carlo Simulation}

In some cases, the noise and interference statistics may be too complicated to obtain a closed-form expression for the moments $M_{\boldsymbol{n}}(\boldsymbol{n})$ or only measurements of $\boldsymbol{n}$ may be available. In those cases, a Monte-Carlo approach may be used to obtain moment estimates

$$
\hat{M}_{n}(L)=\frac{1}{N_{e}} \sum_{k=1}^{N_{e}}\left\|\hat{\boldsymbol{n}}_{k}\right\|^{2 L},
$$

where $\hat{\boldsymbol{n}}_{k}, 1 \leq k \leq N_{e}$, are $N_{e}$ realizations of $\boldsymbol{n}$ which may be obtained by simulation or measurement. The estimates $\hat{M}_{\boldsymbol{n}}(L)$ and $\hat{M}_{\tilde{\boldsymbol{n}}}(L)$ may then be used in Eqs. (12), (14), and (15), respectively, instead of the true moments. This constitutes a semi-analytical approach to asymptotic SEP analysis. We note that this semi-analytical approach is much faster than Monte-Carlo simulation of the SEP since $\hat{M}_{\boldsymbol{n}}(L)$ and $\hat{M}_{\tilde{\boldsymbol{n}}}(L)$ have to be calculated only once and are valid for all SNR values, whereas the SEP has to be simulated for each SNR value separately.

\section{$5 \quad$ Numerical Results and Discussions}

In this section, we verify the derived analytical expressions for the asymptotic BEP and SEP for several practically relevant cases with computer simulations. For calculation of the asymptotic BEP and SEP we used Eqs. (12), (14)-(16) and the required moments were obtained using the methods presented in Section 4. In all figures, we show the BEPs and SEPs as functions of the bit or symbol $\mathrm{SNR}^{5}$ per branch.

BPSK vs. BFSK: Fig. 1 shows the BEPs of BPSK with MRC and BFSK with NC over an i.i.d. Rayleigh fading channel $(L=3)$ impaired by AWGN, i.i.d. $\epsilon$-mixture noise $(\epsilon=0.25, \kappa=10)$, and an i.i.d. Ricean faded $M-\mathrm{PSK}$ interferer with Ricean factor $K_{I}=6 \mathrm{~dB}$ (cf. E5), respectively. For all considered cases the simulation points quickly approach the asymptotic BEP curves as the SNR increases. Furthermore, Fig. 1 confirms that the $6 \mathrm{~dB}$ asymptotic performance loss of NC compared to MRC is independent of the type of noise, cf. Section 3.3.

Spatially dependent $\epsilon$-mixture noise (E8): In Fig. 2, we consider the BEP of 8-PSK with MRC and differential EGC for $L=1,2$, and 3 over an i.i.d. Ricean fading channel ( $K=3 \mathrm{~dB})$ impaired

\footnotetext{
${ }^{5}$ Since, in this paper, we refer to any additive impairment as "noise", we use the term "SNR" even if the received signal is only impaired by what is traditionally referred to as "interference".
} 
by spatially dependent $\epsilon$-mixture noise $(\epsilon=0.25, \kappa=10)$. The simulation results nicely confirm our asymptotic analysis also for spatially dependent noise. Furthermore, Fig. 2 shows that the asymptotic performance loss of differential EGC compared to MRC is, respectively, $3 \mathrm{~dB}, 2.3 \mathrm{~dB}$, and $2 \mathrm{~dB}$ for $L=1,2$, and 3, which is in perfect agreement with the results obtained from Eq. (37).

Asynchronous co-channel interference (E7): Fig. 3 shows the SEP of 4-PSK with MRC over a correlated Rayleigh fading channel $(L=3)$ impaired by a single asynchronous 4-PSK co-channel interferer which also experiences correlated Rayleigh fading. The correlation matrix $C_{h h}$ of the desired user is a Toeplitz matrix with vector $\left[\begin{array}{lll}1 & \alpha & \alpha^{2}\end{array}\right]$ as its first row, where $\alpha$ is the correlation coefficient. The correlation matrix $C_{g g}$ of the interferer has the same structure and its correlation coefficient is denoted by $\rho$. Both the desired user and the interferer employ square-root raised cosine filters with roll-off factor 0.22 for transmit pulse shaping and as receiver input filters. The timing offset between the desired user and the interferer is $\tau=T / 4$. Fig. 3 confirms that the performance of the desired user is not only negatively affected if its own channel is correlated but also if the interference channel is correlated, cf. Section 4.2, E7).

Synchronous co-channel interference (E7): In Fig. 4, we show the SEP of 16-PSK with MRC and differential EGC for correlated Ricean fading $(K=3 \mathrm{~dB})$ and impairment by a correlated Rayleigh faded synchronous 16-PSK co-channel interferer. For $L=3$ the correlation matrices $\boldsymbol{C}_{h h}$ and $\boldsymbol{C}_{g g}$ of the desired user and the interferer, respectively, have the same structure as the corresponding matrices for Fig. 3 with $\alpha=\rho=0.6$. For $L=2$ these matrices are also Toeplitz matrices with $\left[\begin{array}{ll}1 & \alpha\end{array}\right]$ and $\left[\begin{array}{ll}1 & \rho\end{array}\right]$ as first rows, respectively, and the same values for $\alpha$ and $\rho$ are valid as for the $L=3$ case. From Fig. 4 we observe that for the considered type of interference the performance loss of differential EGC compared to MRC is $3 \mathrm{~dB}, 3.9 \mathrm{~dB}$, and $4.3 \mathrm{~dB}$ for $L=1,2$, and 3, respectively, which is in perfect agreement with the values obtained from Eq. (34).

Correlated Gaussian interference (E6): In Fig. 5, we consider the SEP of 4-PSK with MRC over an i.i.d. Rayleigh fading channel with zero-mean correlated Gaussian interference. The interference correlation matrix $C_{n n}$ has the same structure as $C_{g g}$ for Fig. 3 and asymptotic results for $\rho=0$, 0.5 , and 1.0 are shown. For clarity simulation results are only included for $\rho=0.5$. As expected from the discussion in Section 4.2 E6), $\rho=0$ and $\rho=1.0$ result in lower and upper bounds for the SEP achievable for other values of $\rho$. We note that while fully correlated noise $(\rho=1.0)$ results 
in the worst performance, it does not cause a loss in diversity gain. A loss in diversity gain would result of course if the diversity branches of the desired user were fully correlated.

UWB interference: In Fig. 6, we consider the SEP of a 16-QAM narrowband (NB) signal with MRC over i.i.d. Rayleigh fading $(L=2)$ and impairment by direct-sequence UWB (DS-UWB) [18] and multi-band orthogonal frequency multiplexing (MB-OFDM) UWB [19], respectively. The interference channels modeled according to the CM1 UWB channel model [20] and are assumed to be spatially independent. The 16-QAM NB system uses square-root raised cosine filters with roll-off factor 0.22 for transmit pulse shaping and receive filtering. NB system bandwidths of $B=1$ $\mathrm{MHz}$ and $B=5 \mathrm{MHz}$ are considered. Since derivation of a closed-form solution for $M_{\boldsymbol{n}}(L)$ for the relatively complicated UWB signal and channel models does not seem to be feasible, we used the Monte-Carlo method discussed in Section 4.3 to obtain an estimate $\hat{M}_{\boldsymbol{n}}(L)$. This estimate was used subsequently in Eq. (12) to calculate the asymptotic SEP. As can be observed from Fig. 6, the results obtained with this semi-analytical method are in excellent agreement with the simulation results at high SNR. Furthermore, Fig. 6 shows that for the considered scenario the SEP of the NB signal shows a stronger dependence on the NB signal bandwidth $B$ for MB-OFDM UWB interference than for DS-UWB interference. However, for both considered values of $B$, DS-UWB interference is more harmful to the SEP performance of the NB system than MB-OFDM UWB.

\section{Conclusions}

In this paper, we have presented simple, easy-to-evaluate, and insightful asymptotic BEP and SEP expressions for quadratic diversity combining receivers operating in correlated Ricean fading and non-Gaussian noise and interference. The only assumption necessary for the validity of the presented results is that all joint noise moments exist. We could show that while the diversity order of the considered combining schemes is independent of the type of noise, their combining gain is affected by the type of noise via certain noise moments. We have provided general techniques for calculation of these noise moments and we have tabulated the moments of many practically relevant types of noise. Our analytical results show that not only fading correlation but also noise correlation negatively affects the performance of diversity combiners. Furthermore, while BFSK with NC suffers 
from an asymptotic performance loss of $6 \mathrm{~dB}$ compared to BPSK with MRC regardless of the type of noise, the performance loss of differential EGC compared to MRC crucially depends on the type of noise if more than one diversity branches are available.

\section{References}

[1] M.K. Simon and M.-S. Alouini. Digital Communication over Fading Channels. Wiley, Hoboken, New Jersey, 2005.

[2] J.G. Proakis. Digital Communications. McGraw-Hill, New York, forth edition, 2001.

[3] H. Abdel-Ghaffar and S. Pasupathy. Asymptotic Performance of $M$-ary and Binary Signals Over Multipath/Multichannel Rayleigh and Ricean Fading. IEEE Trans. Commun., COM-43:27212731, November 1995.

[4] Z. Wang and G.B. Giannakis. A Simple and General Parameterization Quantifying Performance in Fading Channels. IEEE Trans. Commun., COM-51:1389-1398, August 2003.

[5] M. Win, N. Beaulieu, L. Shepp, B. Logan, and J. Winters. On the SNR Penalty of MPSK with Hybrid Selection/Maximal Ratio Combining over I.I.D. Rayleigh Fading Channels. IEEE Trans. Commun., 51:1012-1023, June 2003.

[6] Z. Du, J. Chen, and N. Beaulieu. Asymptotic BER Performance of OFDM in FrequencySelective Nakagami- $m$ Channels. In Proceedings of the IEEE Vehicular Technology Conference (VTC), pages 612-615, September 2004.

[7] Y. Ma, Z. Wang, and S. Pasupathy. Asymptotic Performance of Hybrid-Selection/MaximalRatio Combining over Fading Channels. IEEE Trans. Commun., COM-54:770-777, May 2006.

[8] A. Shah and A. Haimovich. Performance Analysis of Maximal Ratio Combining and Comparison with Optimum Combining for Mobile Radio Communications with Cochannel Interference. IEEE Trans. Veh. Technol., COM-49:1454-1463, July 2000.

[9] C. Tepedelenlioglu and P. Gao. On Diversity Reception Over Fading Channels with Impulsive Noise. IEEE Trans. Veh. Technol., 54:2037-2047, November 2005.

[10] C.A. Corral, S. Emami, and G. Rasor. Model of Multi-Band OFDM Interference on Broadband QPSK Receivers. In Proceedings of the IEEE International Conference on Acoustics, Speech, and Signal Processing (ICASSP), pages 629-632, Philadelphia, November 2005.

[11] A. Nasri, R. Schober, and Y. Ma. Unified Asymptotic Analysis of Linearly Modulated Signals in Fading, Non-Gaussian Noise, and Interference. Revised paper submitted to the IEEE Transaction on Communications, October 2006.

[12] M. Schwartz, W. Bennett, and S. Stein. Communication Systems and Techniques. McGrawHill, New York, 1966.

[13] B. Picinbono. On Circularity. IEEE Trans. Signal Processing, 42:3473-3482, December 1994.

[14] M. Abramowitz and I. Stegun. Handbook of Mathematical Functions. Dover Publications, Inc., New York, 1970.

[15] D. Middleton. Statistical-physical Models of Man-made Radio Noise - Parts I and II. U.S. Dept. Commerce Office Telecommun., April 1974 and 1976.

[16] J. Cui and A. Sheikh. Outage Probability of Cellular Radio Systems Using Maximal Ratio Combining in the Presence of Multiple Interferers. IEEE Trans. Commun., 47:1121-1124, August 1999. 
[17] X. Zhang and N. Beaulieu. Outage Probability of MRC With Unequal-Power Cochannel Interferers in Correlated Rayleigh Fading. IEEE Commun. Letters, 10:7-9, January 2006.

[18] IEEE P802.15. DS-UWB Physical Layer Submission to IEEE 802.15 Task Group 3a (Doc. Number P802.15-03/0137r4). January 2005.

[19] IEEE P802.15. Multiband OFDM Physical Layer Proposal for IEEE 802.15 Task Group 3a (Doc. Number P802.15-03/268r3). March 2004.

[20] A. Molisch, J. Foerster, and M. Pendergrass. Channel Models for Ultrawideband Personal Area Networks. IEEE Wireless Communications, 10:14-21, dec 2003. 


\section{Figures and Tables:}

Table 1: Parameters $\beta_{M}$ and $d_{M}$ for various signal constellations $\mathcal{A}$.

\begin{tabular}{|l|c|c|}
\hline Modulation Scheme & $\beta_{M}$ & $d_{M}$ \\
\hline \hline$M-\mathrm{PAM}$ & $2\left(1-\frac{1}{M}\right)$ & $2 \sqrt{\frac{3}{M^{2}-1}}$ \\
\hline $\operatorname{BPSK}(M=2)$ & 1 & 2 \\
\hline$M-\mathrm{PSK}(M \geq 4)$ & 2 & $2 \sin \left(\frac{\pi}{M}\right)$ \\
\hline$M$-QAM & $4\left(1-\frac{1}{\sqrt{M}}\right)$ & $\sqrt{\frac{6}{M-1}}$ \\
\hline
\end{tabular}


Table 2: Moments $M_{n}(N)=\mathcal{E}\left\{|n|^{2 N}\right\}$ of scalar Gaussian RVs and the RVs discussed in Examples E1)-E3). Gaussian RV: Mean $\mu_{n}$ and variance $\sigma_{n}^{2}$. The parameters for the other RVs are defined in Section 4.1.1.

\begin{tabular}{|c|l|}
\hline Scalar Noise Model & Moments $M_{n}(N)$ \\
\hline \hline Gaussian RV $\left(\mu_{n}=0\right)$ & $M_{n}(N)=N ! \sigma_{n}^{2 N}$ \\
\hline Gaussian RV $\left(\mu_{n} \neq 0\right)$ & $M_{n}(N)=N !{ }_{1} F_{1}\left(-N, 1 ;-\left|\mu_{n}\right|^{2} / \sigma_{n}^{2}\right) \sigma_{n}^{2 N}$ \\
\hline Gaussian Mixture & $M_{n}(N)=N ! \sum_{k=1}^{I} c_{k} \sigma_{k}^{2 N}$ \\
\hline $\begin{array}{c}M_{i} \text {-ary Interference } \\
\text { with Fixed Channel }\end{array}$ & $M_{n}(N)=\frac{1}{M_{i}^{k_{1}-k_{l}+1}} \sum_{n_{0} \in \mathcal{S}}\left|n_{0}\right|^{2 N}$ \\
\hline $\begin{array}{c}M_{i}-\text { PSK Interference } \\
\text { with Random CP }\end{array}$ & $M_{n}(N)=|g|^{2 N}$ \\
\hline
\end{tabular}


Table 3: Moments $M_{\boldsymbol{n}}(L)=\mathcal{E}\left\{|\boldsymbol{n}|^{2 L}\right\}$ of RVVs. Zero-mean i.i.d. Gaussian RVV: Variance of each element $\sigma_{n}^{2}$. Correlated Gaussian RVV: $\lambda_{l}, 1 \leq l \leq L$, are the eigenvalues of covariance matrix $\boldsymbol{C}_{n n} \triangleq \mathcal{E}\left\{\boldsymbol{n} \boldsymbol{n}^{H}\right\}$. The parameters of the Gaussian mixture RVV are defined in Section 4.2, Example E9).

\begin{tabular}{|c|l|}
\hline Vector Noise Model & Moments $M_{\boldsymbol{n}}(L)$ \\
\hline \hline I.I.D. Gaussian RVV & $M_{\boldsymbol{n}}(L)=\frac{(2 L-1) !}{(L-1) !} \sigma_{n}^{2 L}$ \\
\hline Correlated & $M_{\boldsymbol{n}}(L)=L ! \sum_{k_{1}+\ldots+k_{L}=L} \lambda_{1}^{k_{1}} \cdot \ldots \cdot \lambda_{L}^{k_{L}}$ \\
Gaussian RVV & $M_{\boldsymbol{n}}(L)=\frac{(2 L-1) !}{(L-1) !} \sum_{k=1}^{I} c_{k} \sigma_{k}^{2 L}$ \\
\hline $\begin{array}{c}\text { Spatially Dependent } \\
\text { Gaussian Mixture }\end{array}$ \\
\hline
\end{tabular}




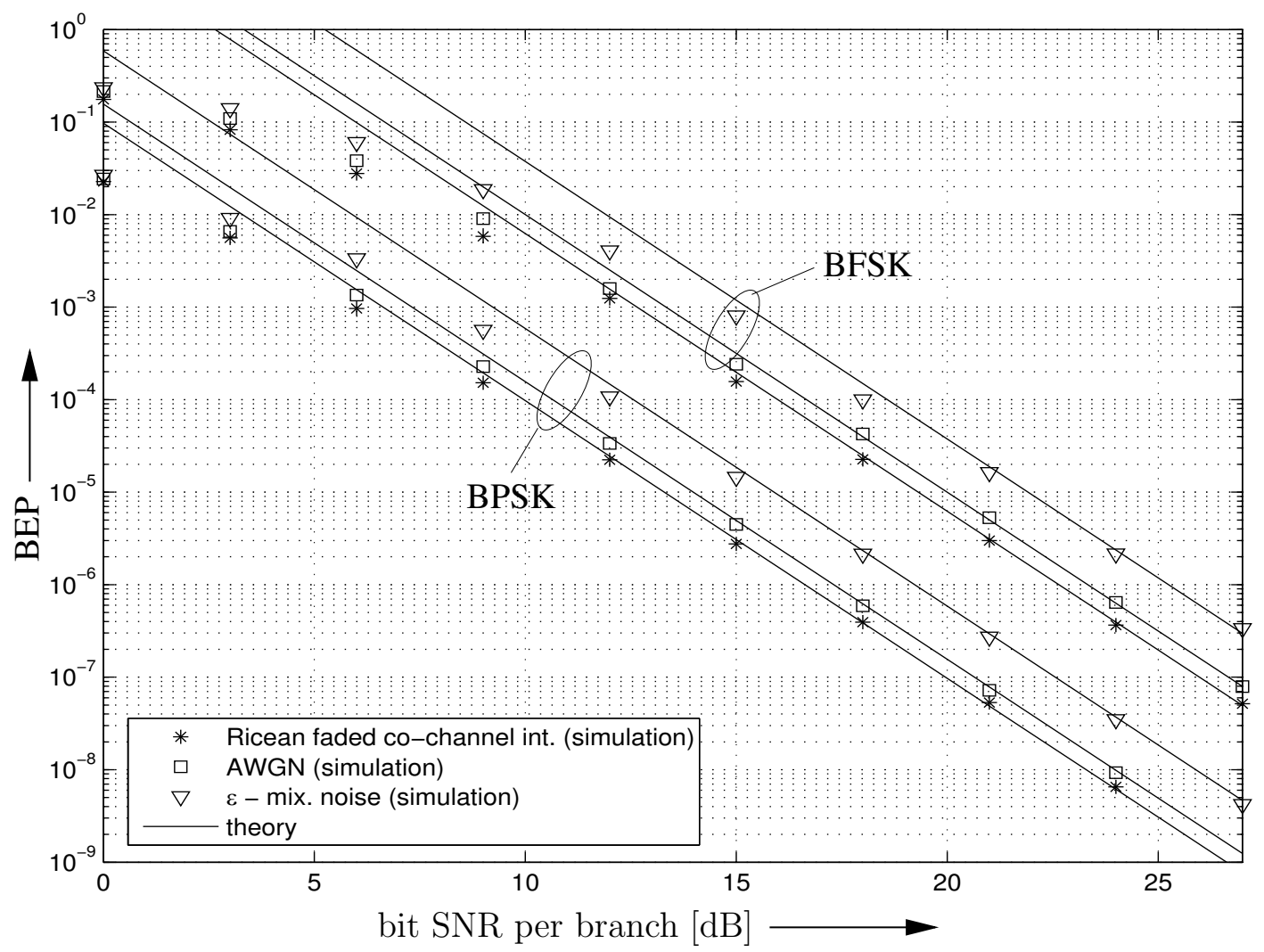

Figure 1: BEP of BPSK with MRC and BFSK with NC vs. bit SNR per branch for i.i.d. Rayleigh fading and $L=3$. Impairment by AWGN, i.i.d. $\epsilon-$ mixture noise $(\epsilon=0.25, \kappa=10)$, and a Ricean faded $M-\mathrm{PSK}$ interferer with Ricean factor $K_{I}=6$ dB (cf. E5). Markers: Simulated BEP. Solid lines: Asymptotic BEP [Eqs. (12), (15), and (16)]. 


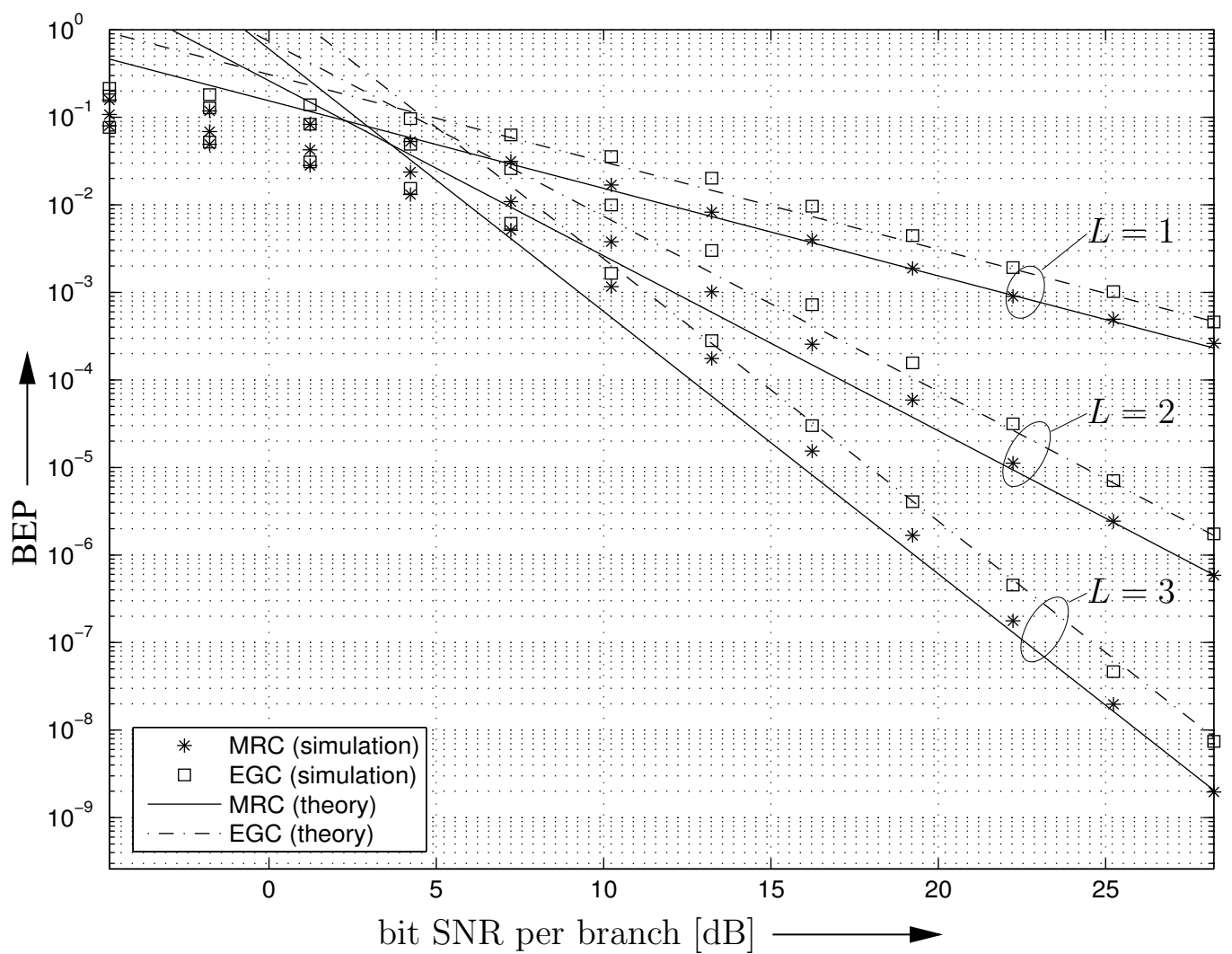

Figure 2: BEP of 8-PSK vs. bit SNR per branch for differential EGC and MRC over an i.i.d. Ricean fading channel with Ricean factor $K=3 \mathrm{~dB}$ and spatially dependent $\epsilon-$ mixture noise $(\epsilon=0.25, \kappa=10)$. Markers: Simulated BEP. Lines: Asymptotic BEP [Eqs. (12), (14), and (16)]. 


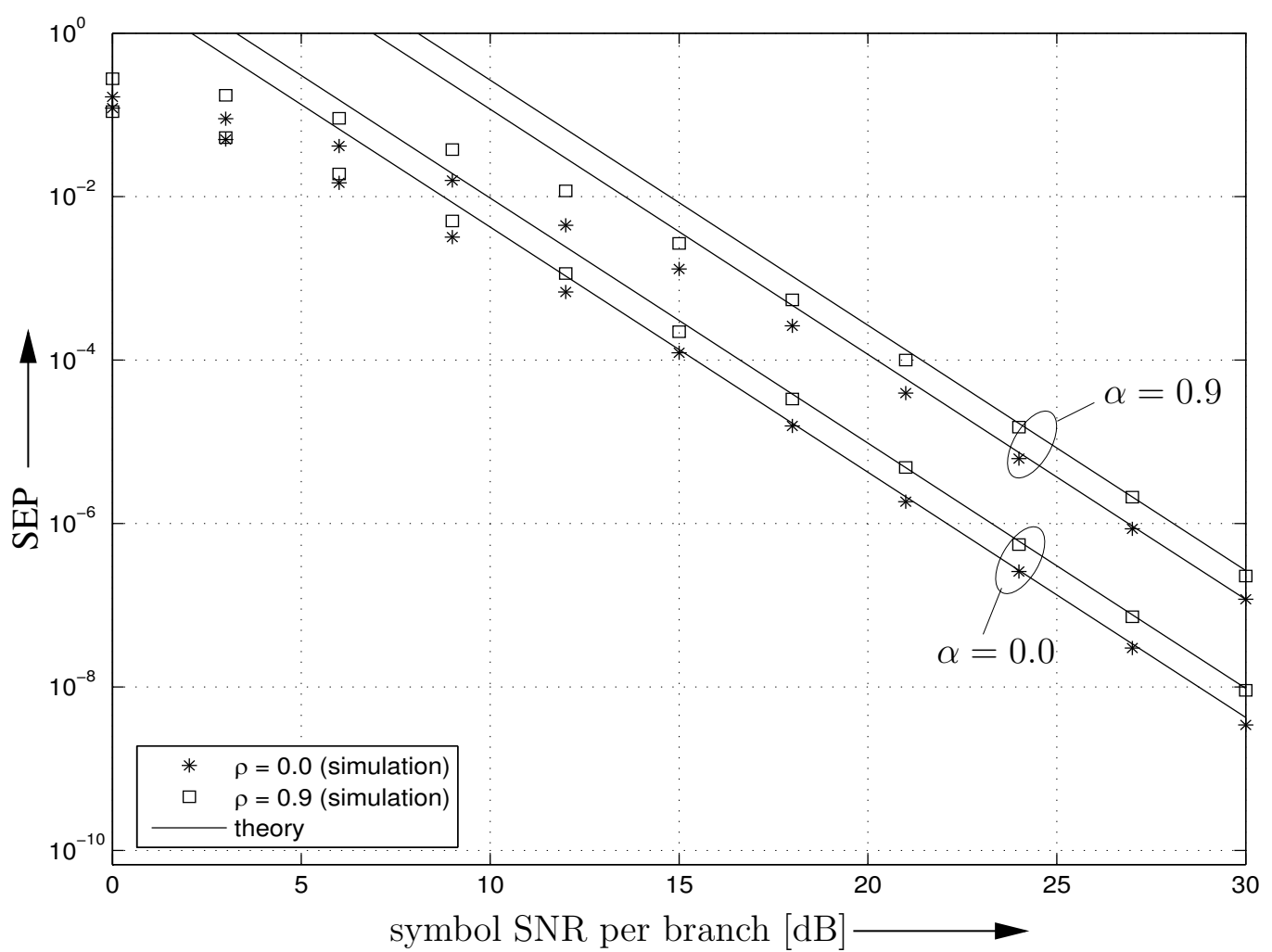

Figure 3: SEP of 4-PSK vs. symbol SNR per branch for MRC over uncorrelated $(\alpha=0.0)$ and correlated $(\alpha=0.9)$ Rayleigh fading channels $(L=3)$ with uncorrelated $(\rho=0.0)$ and correlated $(\rho=0.9)$ Rayleigh faded asynchronous $4-\mathrm{PSK}$ co-channel interference. Markers: Simulated SEP. Solid lines: Asymptotic SEP [Eq. (12)]. 


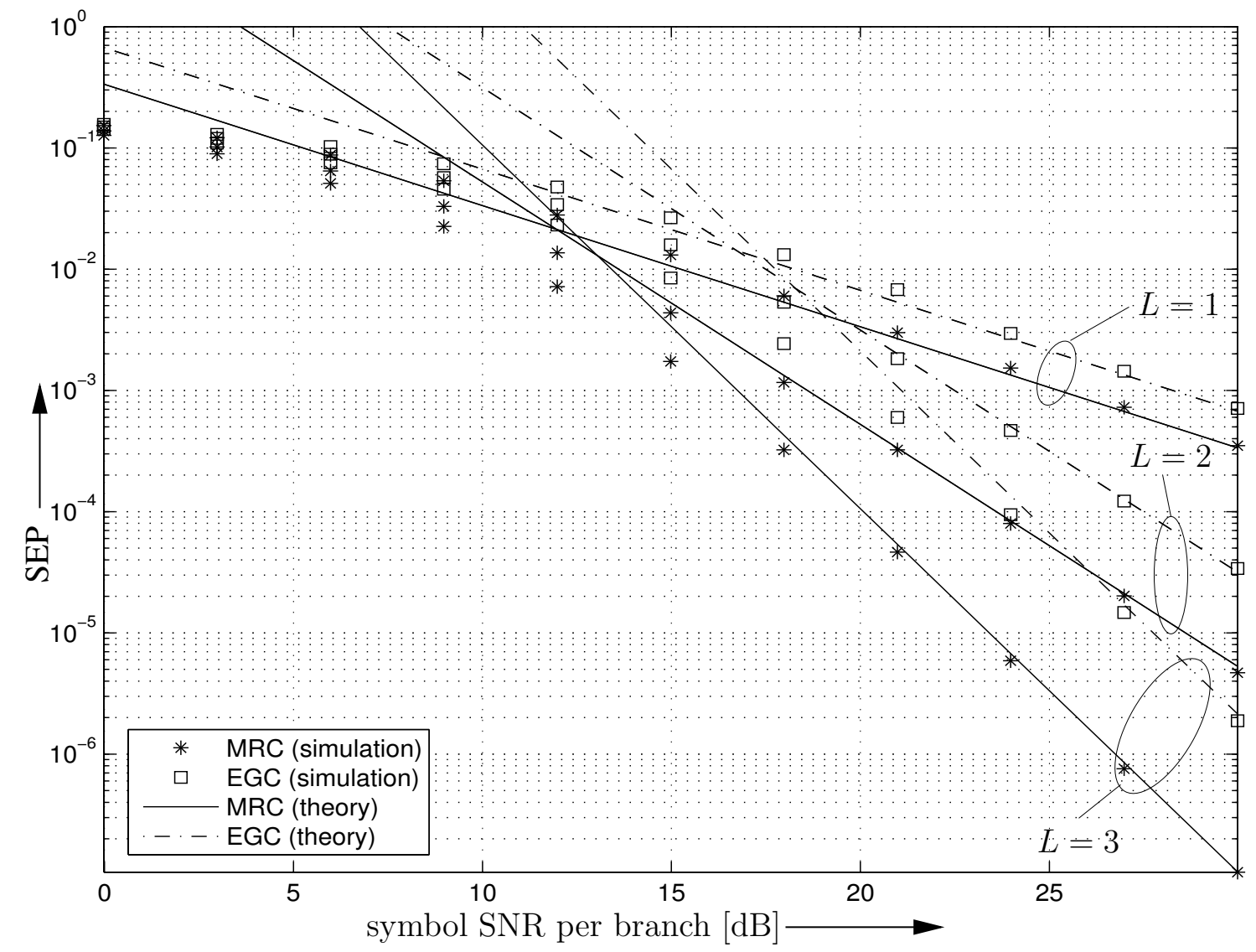

Figure 4: SEP of 16-PSK vs. symbol SNR per branch for MRC and differential EGC over correlated Ricean fading channels ( $K=3 \mathrm{~dB}, \alpha=0.6)$ with correlated Rayleigh faded co-channel interference $(\rho=0.6)$. Markers: Simulated SEP. Lines: Asymptotic SEP [Eq. (12), (14)]. 


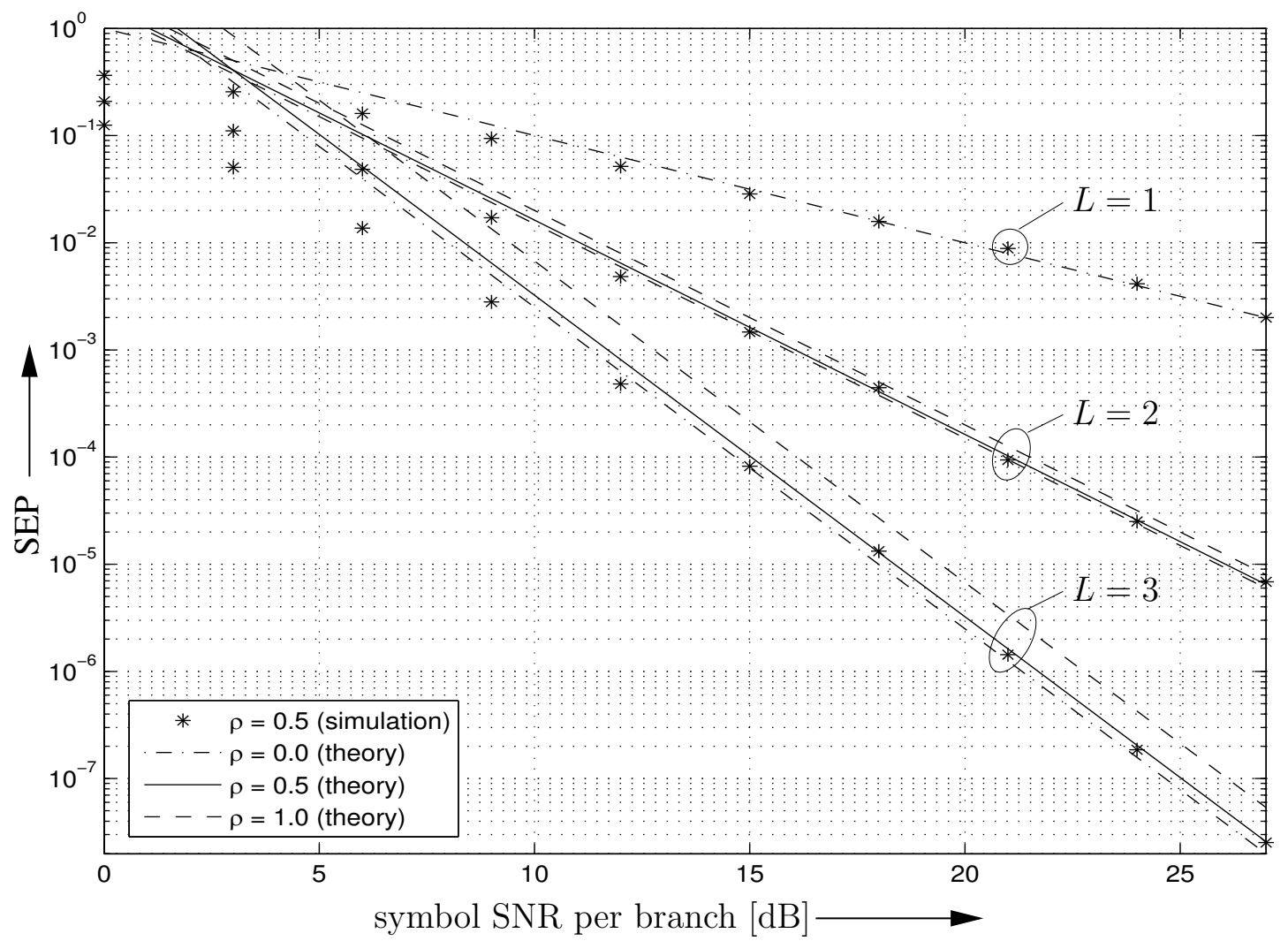

Figure 5: SEP of 4-PSK vs. symbol SNR per branch for MRC over i.i.d. Rayleigh fading channels with zero-mean correlated Gaussian interference. Markers: Simulated SEP. Lines: Asymptotic SEP [Eq. (12)]. 


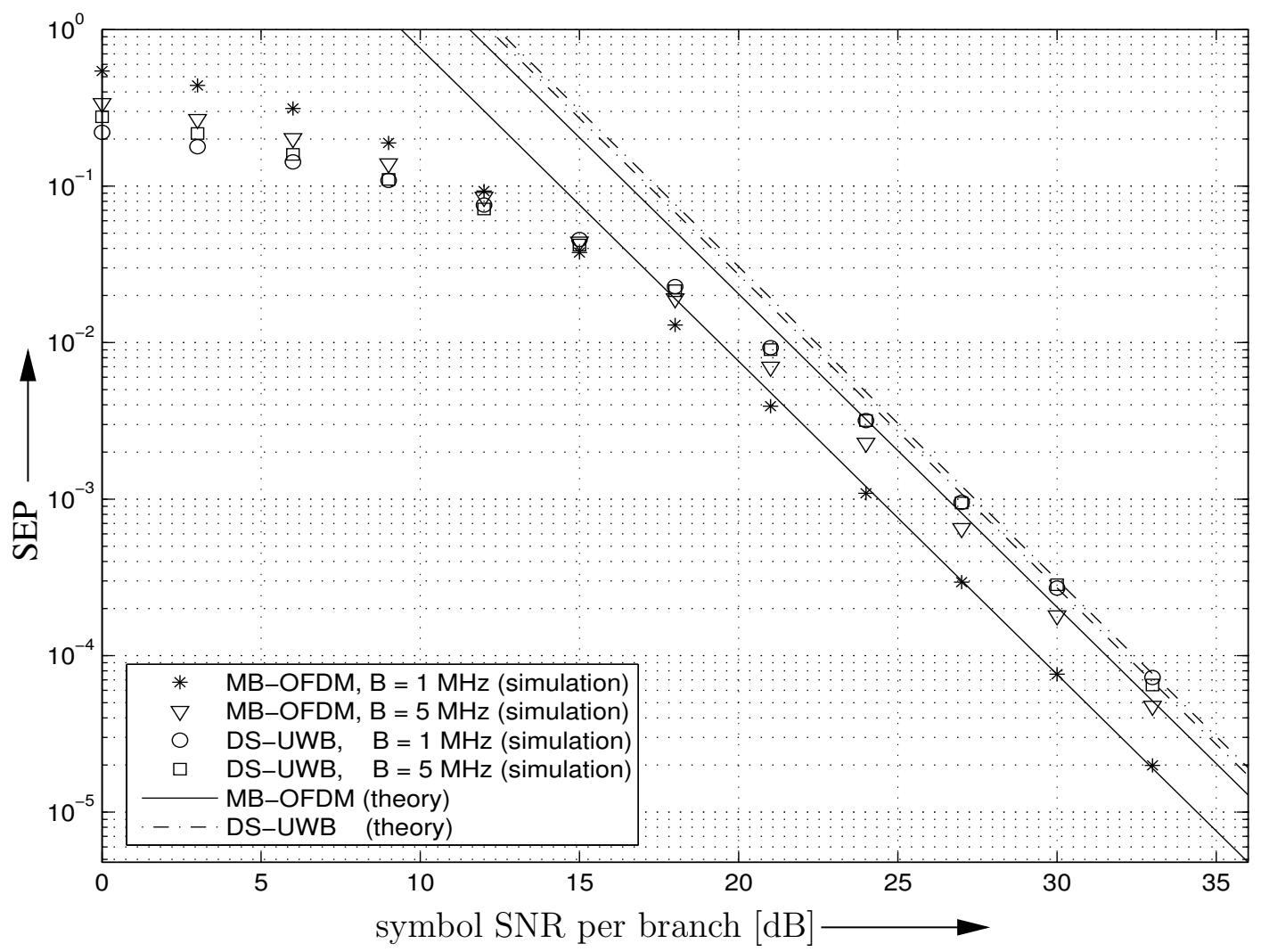

Figure 6: SEP of 16-QAM vs. symbol SNR per branch for MRC over i.i.d. Rayleigh fading channels $(L=2)$ with DS-UWB and MB-OFDM UWB interference. Markers: Simulated SEP. Lines: Asymptotic SEP [Eq. (12)]. 